\title{
Concerning: "The sad plight of multiple sclerosis research (low on fact, high on fiction): critical data to support it being a neurocristopathy" by Peter O. Behan and Abhijit Chaudhuri
}

\author{
Adrian R. M. Upton
}

Received: 11 October 2010/ Accepted: 12 October 2010/Published online: 3 November 2010

(C) Springer Basel AG 2010

Professor Peter Behan and Dr. Abhijit Chaudhuri criticize the current state of multiple sclerosis (MS) research and treatment. They propose that MS is a developmental neural crest disorder (a cristopathy) with glial cell dysfunction and diffuse breakdown of the blood-brain barrier.

The authors take issue with the claim that MS is an autoimmune disorder as it is a heterogeneous condition that defies both clinical and exact definition. Assumptions about an autoimmune or viral aetiology have not been supported by firm reproducible evidence. Concentration on an autoimmune basis for MS may have delayed development of useful treatments of MS to slow or prevent disease progression.

Professor Behan discusses genetic factors and associated disorders such as neurofibromatosis, glioma, glioblastoma multiforme and Charcot-Marie-Tooth peripheral neuropathy with possible links to chromosome $17 \mathrm{q}$ and neural crest disorders.

The current state of treatment with $\beta$ interferon is limited by modest efficacy and a lack of understanding how $\beta$ interferon may work. Immunosuppressive therapy has not provided a cure and it can be associated with serious side effects and complications, including fatality. There is no immunological test that is diagnostic of MS and immunotherapy has about a $30 \%$ effect in relapsing/remitting MS within a placebo effect.

Pregnancy can stabilize the clinical course of MS whereas a classical autoimmune disease (systemic lupus erythematosus) often flares up during pregnancy. The reliance on MRI

A. R. M. Upton ( $\square)$

Division of Neurology, Department of Medicine,

McMaster Medical Centre, 4U8-1200 Main Street West,

Hamilton, ON L8N 3Z5, Canada

e-mail: uptona@hhsc.ca studies as showing inflammatory lesions is limited by the lack of evidence that MS plaques reflect specific $\mathrm{T}$ cell mediated autoimmune inflammatory processes. A third of plaques show no inflammatory infiltrate and other plaques show mild or inconsistent mononuclear infiltrates.

There is an extensive review of literature, but in some areas it is selective as in the discussion of trauma, electrical injury and MS.

Reliance on experimental allergic encephalomyelitis (EAE) as a reliable animal model of human MS is not based on sound scientific rationale and there is an extensive discussion of this issue.

The authors claim that the 8 billion dollars (US) MS market has encouraged commercialization of clinical research for the benefit of all, but the patients. There are nearly 40,000 patients receiving natalizumab at an annual cost of $\$ 25,000$ (US) without long-term evidence that it prevents disability progression.

The authors recommend research in the area of neural crest associations.

This is a refreshing and thought provoking article that questions current concepts of MS research and treatment. The minimal progress in treatment of MS over the last 100 years has encouraged the appearance of multiple bogus remedies (diet, Russell viper venom, hyperbaric chamber, vein surgery) that appear attractive to desperate patients and their families.

It is to be hoped that this article will encourage a more comprehensive program of investigation leading to better understanding of MS and significant effects on disease progression. 